\title{
LOS LÍMITES A LA APERTURA DEL SISTEMA AUTONÓMICO EN EL INFORME SOBRE LA REFORMA DE LA CONSTITUCIÓN
}

\author{
MARÍA SALVADOR MARTÍNEZ \\ Profesor Ayudante de Derecho Constitucional \\ Universidad Nacional de Educación a Distancia
}

\author{
SUMARIO \\ I. El Informe sobre la reforma de la Consti- \\ tución Española. \\ II. La delimitación de la consulta relativa a la \\ denominación de las Comunidades Au- \\ tónomas. \\ III. El principio dispositivo y la apertura del \\ sistema autonómico. \\ IV. Los límites del sistema, y las leyes de \\ transferencia y delegación.
}

A raíz de la iniciativa anunciada por el Gobierno de modificar la Constitución para incluir la denominación de las Comunidades Autónomas y de los últimos procesos de reforma de Estatutos de Autonomía, se ha intensificado el debate sobre el grado de apertura de nuestro sistema de distribución territorial del poder. El Consejo de Estado, consultado en su día por el Gobierno sobre la reforma del texto constitucional para incluir la denominación de las Comunidades Autónomas, ha contribuido a dicho debate pronunciándose sobre la apertura del sistema autonómico, los límites a la misma y ciertas modificaciones relativas a dicha apertura que contribuirían a completar y perfeccionar la reforma proyectada por el Gobierno. El objeto de estas líneas es analizar brevemente el apartado del Informe del Consejo de Estado sobre la reforma de la Constitución Española que se dedica a la apertura del sistema autonómico y al papel que juegan las leyes orgánicas de transferencia y delegación en la apertura de dicho sistema. 


\section{EL INFORME SOBRE LA REFORMA DE LA CONSTITUCIÓN}

Como se sabe, a principios de 2005 el Gobierno remitió una consulta al Consejo de Estado solicitándole un informe sobre cuatro modificaciones constitucionales que se había propuesto llevar a cabo: la supresión de la preferencia del varón en la sucesión al trono, la recepción en la Constitución del proceso de construcción europea, la inclusión de la denominación de las Comunidades Autónomas y la reforma del Senado ${ }^{1}$. De acuerdo con el nuevo régimen jurídico del Consejo de Estado ${ }^{2}$, la Comisión de Estudios del mismo comenzó sus trabajos el 9 de marzo de ese año, creando un Grupo de trabajo que se ocuparía de cada una de las cuatro cuestiones planteadas por el Gobierno, y, finalizada su labor, en la sesión plenaria del Consejo de 16 de febrero, por 24 votos a favor y uno en contra, se aprobó el "Informe sobre modificaciones de la Constitución Española", al que se sumaron un voto separado coadyuvante con la mayoría y dos votos particulares ${ }^{3}$.

Respecto de cada una de las cuatro modificaciones constitucionales propuestas por el Gobierno, en la consulta al Consejo de Estado se justificaba la necesidad de la reforma, se señalaban los objetivos a alcanzar, se ofrecían ciertos criterios sobre la misma y se especificaba exactamente qué se solicitaba del Consejo mediante la formulación de preguntas concretas sobre cada uno de los cuatro temas. No obstante, el Gobierno precisó en la misma consulta que "todo ello sin perjuicio de que el Consejo de Estado aborde el estudio de otros aspectos de las mencionadas modificaciones o estrechamente relacionadas con ellas que considere conveniente tener en cuenta para completarlas o mejorar su calidad técnican. ${ }^{4}$ La consulta se formulaba así en términos coherentes con lo previsto en la Ley Orgánica del Consejo de Estado, de acuerdo con la cual «en la elaboración de las propuestas legislativas o de reforma constitucional (el Consejo de Estado) atenderá los objetivos, criterios y límites de la refor-

1 Acuerdo del Consejo de Ministros de 4 de marzo de 2005. Textualmente, las cuatro cuestiones son: «la modificación, sin alterar las previsiones que afectan al Príncipe de Asturias, de las normas que regulan el orden de sucesión en la corona, con el fin de adaptarlas al principio de no discriminación de la mujer; la incorporación del compromiso asumido por los ciudadanos españoles con el proceso de construcción política de Europa; la recepción constitucional de la denominación oficial de las Comunidades Autónomas y de las Ciudades Autónomas; y la reforma del Senado para hacer de esta Cámara lo que la propia Constitución define y proyecta para la misma, el espacio institucional para la defensa de la identidad, autogobierno y participación de las Comunidades Autónomas, reforzando la cohesión y la colaboración en una tarea común".

2 El régimen jurídico del Consejo de Estado se modificó por la Ley 3/2004, de 28 de diciembre, entre otras cosas para hacer posible la consulta del Gobierno al Consejo sobre esta reforma constitucional. Vid. M. SALVADOR MARTÍNEZ, "El Consejo de Estado ante la reforma constitucional, en Teoría y Realidad Constitucional, n. ${ }^{\circ} 16,2005$, pp. 467 y ss.

3 El texto del informe puede consultarse en www.consejo-estado.es (este es el texto del informe que aquí citamos); también en F. RUBIO LLORENTE Y J. ÁLVAREZ JUNCO (eds.), El informe del Consejo de Estado sobre la reforma constitucional. Texto del informe y debates académicos, Consejo de Estado-CEPC, Madrid, 2006, pp. 51 y ss.

4 Consulta del Gobierno al Consejo de Estado, p. 39. 
ma constitucional señalados por el Gobierno, y podrá hacer también las estimaciones que estime pertinentes acerca de ellos"s.

En la introducción al Informe sobre modificaciones de la Constitución Española, el Consejo de Estado se refiere al contenido y estructura del mismo. Respecto al contenido, afirma que éste debe situarse dentro del marco establecido por la consulta, centrarse en el estudio de los cuatro puntos cuya modificación se proyecta y respetar las condiciones a las que a juicio del Gobierno ha de sujetarse la reforman. ${ }^{6}$ En cuanto a la estructura del Informe, éste se ordena en torno a cuatro partes, cada una dedicada a una de las cuestiones planteadas por el Gobierno, y en cada una de ellas se responde a las preguntas concretas formuladas en la consulta, sugiriendo redacciones posibles de los preceptos que han de modificarse según las distintas alternativas que se ofrecen. No obstante, apoyándose en la posibilidad que le ofreció el Gobierno de abordar el estudio de otros aspectos que el Consejo considerase conveniente, y en la misma posibilidad que le permite la Ley Orgánica del Consejo de Estado, en el Informe se ha incluido algo más que la respuesta concreta a las preguntas planteadas. Salvo en la primera parte, dedicada a la supresión de la preferencia del varón en la sucesión al trono, en todas las demás "se ha agregado un apartado final que incluye el estudio de otras cuestiones estrechamente relacionadas con dichas modificaciones, que no es indispensable tomar en cuenta para alcanzar los objetivos que la reforma se propone, pero que podrían completarla y perfeccionarla"; en relación a estas cuestiones conexas "el Consejo de Estado no considera conveniente ofrecer, ni siquiera como ejemplo, la eventual redacción que haya de darse a los preceptos correspondientes". ${ }^{7}$

Como reconoce el prof. Rubio Llorente, Presidente del Consejo de Estado, de la Comisión de Estudios y Director del Grupo de Trabajo n. ${ }^{\circ}$ 3, encargado de la inclusión de la denominación de las Comunidades Autónomas en la Constitución, «el capítulo del informe en el que estas consideraciones complementa-

5 Art. 2.3 LO 3/1980, de 22 de abril, del Consejo de Estado.

6 Consejo de Estado, Informe sobre modificaciones de la Constitución Española, p. 13. Las condiciones que el Consejo de Estado deduce de la consulta son las siguientes. Primera, que ninguna de las modificaciones proyectadas pretende rectificar o invertir el núcleo de las decisiones adoptadas en su día por el constituyente, sino que buscan completar y culminar en cada caso, adaptándolo al presente, el diseño normativo de 1978. Segunda, que el éxito de la reforma depende del respeto a "los valores, principios y opciones fundamentales que el constituyente de 1978 estableció como columna vertebral de la Constitución". Tercera, que la función del Consejo de Estado no es "la de valorar los cambios cuya introducción se plantea para asegurar que responden a demandas consistentes y buscan resolver problemas o insuficiencias ampliamente reconocidas, pues tanto la valoración como el juicio son competencia de los poderes políticos". Cuarta, la voluntad del Gobierno "de que los cambios sean limitados y prudentes para no alterar el equilibrio en que se sustenta el texto constitucional". Y quinta, que, aunque no está al alcance del Consejo de Estado "lograr que las alternativas propuestas hayan sido suficientemente maduradas y sean consecuencia de un diálogo sostenido y sereno entre las fuerzas políticas y con la sociedad", el Consejo habrá de llevar a cabo su tarea de la manera que resulte más adecuada para el cumplimiento de tales condiciones (pp. 14 a 16).

7 Ibíd., pp. 16 y 17. 
rias se llevan más lejos es el cuarto, es decir, aquél en el que se analiza el propósito de llevar a la Constitución los nombres de las Comunidades Autónomas. La Comisión de Estudios, primero, y el Pleno del Consejo, después, han entendido que éste era, como proponía el Ponente, el lugar adecuado para incorporar, siquiera un esbozo, una reflexión general sobre nuestro peculiar modelo de organización territorial ${ }^{8}$.

\section{LA DELIMITACIÓN DE LA CONSULTA RELATIVA A LA INCLUSIÓN DE LA DENOMINACIÓN DE LAS COMUNIDADES AUTÓNOMAS}

En relación a la propuesta de incluir la denominación de las Comunidades Autónomas en la Constitución, en la consulta del Gobierno al Consejo de Estado se hace un breve repaso del proceso de construcción de nuestra forma de organización territorial para concluir afirmando lo siguiente:

"es tiempo de consolidar los logros alcanzados, reconociendo de forma expresa en nuestra Constitución a los sujetos institucionales que han protagonizado todo ese proceso. ... Designar por su nombre a todas y cada una de las Comunidades Autónomas que integran España no es un innecesario ejercicio de estilo. Por el contrario, significa superar la apertura inicial del modelo de descentralización territorial establecido por el constituyente... En su discurso de investidura el Presidente del Gobierno manifestó su deseo de reformar la Constitución para incluir en la misma una referencia expresa a todas y cada una de las diecisiete Comunidades Autónomas. En Gobierno en consonancia con ese compromiso, solicita del Consejo de Estado que informe sobre esta cuestión, precisando, en todo caso, los siguientes extremos: 1. Qué artículo o artículos de la Constitución son los más idóneos para llevar a cabo esa mención expresa e individualizada de las Comunidades Autónomas y las dos Ciudades Autónomas; 2. Qué criterio o criterios se consideran más adecuados para ordenar su enumeración; 3. Qué consecuencias jurídicas produce la constitucionalización de las Comunidades Autónomas en la Constitución y qué preceptos del texto constitucional convendría modificar para reflejarlas" ${ }^{9}$.

Antes de dar respuesta a estas cuestiones concretas, el Consejo de Estado dedica unas páginas de su Informe a delimitar la cuestión sometida a consulta ${ }^{10}$. Para ello, analiza la consulta del Gobierno y concluye que el objetivo del Gobierno es doble, que "la propuesta acerca de la cual se solicita su informe encierra una doble decisión" ${ }^{11}$.

8 F. RUBIO LLORENTE, y J. ÁLVAREZ JUNCO (eds.), El informe del Consejo de Estado sobre la reforma constitucional. Texto del informe y debates académicos, op. cit., p. 23.

9 Consulta del Gobierno al Consejo de Estado, p. 30.

10 Capítulo IV, Apartado 1.2. del Informe sobre modificaciones de la Constitución Española, pp. 135 a 146.

11 Ibíd., p. 144. 
En primer lugar, al proponer que se mencionen en la Constitución los nombres de las Comunidades Autónomas se trata de "superar la apertura inicial del modelo de descentralización política establecido por el constituyente", consecuencia del llamado principio dispositivo que dejó en manos de los sujetos territorialmente legitimados la determinación del número de Comunidades Autónomas y sus competencias, y que dio lugar a un largo proceso a través del cual se ha ido construyendo nuestro modelo de descentralización política, un modelo "propio y específico" ${ }^{12}$. Ésta sería la "decisión expresa" del Gobierno, "la de superar la apertura inicial del sistema, consagrada en el principio dispositivo, llevando a la Constitución las consecuencias a las que ha dado lugar su aplicación para organizar el territorio nacional en Comunidades Autónomas", y estaría "plasmada en las preguntas explícitas que se formulan al Consejo y que habrán de ser respondidas con la debida precisión, incluso con propuestas específicas de preceptos ${ }^{13}$.

En segundo lugar, deduce el Consejo que el Gobierno también se propone "Consolidar un modelo propio de descentralización política", lo cual implica una matización importante del primer objetivo de "superar la apertura inicial de dicho modelo", pues la característica más destacada del mismo ha sido la de permanecer abierto a impulsos del principio dispositivo ${ }^{14}$. Esta sería la "decisión tácita" del Gobierno, "la de mantener una cierta apertura en el sistema, después de creadas las Comunidades Autónomas, a través de la reforma posible de los Estatutos". Esta segunda decisión "no se concreta en pregunta alguna", pero, a juicio del Consejo, "este Informe quedaría incompleto si no la tuviese presente para formular sobre ella algunas reflexiones — sin sugerencia sobre posibles textos - acogiéndose así a la propia invitación contenida en la consulta del Gobierno" ${ }^{15}$.

De acuerdo con esta delimitación del tema sometido a consulta, de un lado, el Consejo de Estado, en los apartados 2, 3 y 4 del capítulo IV del Informe, ofrece una respuesta concreta a las preguntas que se le formulan, y que responden al primer objetivo del Gobierno. De otro lado, añade un último apartado a ese capítulo IV, en el que, respondiendo al segundo objetivo del Gobierno, "se ocupa de cuestiones estrechamente relacionadas con la reforma proyectada para sugerir cambios que, si se estimasen políticamente posibles y oportunos, contribuirían a completarla y a perfeccionar, en consecuencia, el sistema de distribución territorial del poder establecido por la Constitución, sin alteración alguna de éste " ${ }^{16}$. A la hora de decidir y justificar cuáles son esas otras cuestiones que va a tratar, el Consejo afirma que, aunque las modificaciones propuestas en el Informe como respuesta a las preguntas concretas del Gobierno podrían abrir un abanico muy amplio de cambios complementarios,

12 Ibíd., p. 135.

13 Ibíd., p., 145

14 Ibíd., p. 136.

15 Ibíd., p. 145.

16 Ibíd., pp. 145 y 146. 
debe limitarse a reflexionar sobre las cuestiones cuya relación inmediata y estrecha con las citadas modificaciones sea evidente e incuestionable, y, dentro de ellas, sobre los problemas relacionados con el funcionamiento general del sistema, sin entrar en los que plantean los distintos criterios que la Constitución emplea para la delimitación de competencias. De acuerdo con esto, las tres cuestiones seleccionadas son: los principios constitucionales que rigen la relación de las Comunidades Autónomas entre sí y con el Estado (solidaridad, igualdad, cooperación y colaboración); la relación entre las Comunidades Autónomas y las provincias; y el mecanismo que asegura la apertura del sistema ${ }^{17}$. Esta última cuestión es la que aquí nos interesa.

\section{EL PRINCIPIO DISPOSITIVO Y LA APERTURA DEL SISTEMA AUTONÓMICO}

Como se sabe, los constituyentes de 1978, habiendo heredado un Estado fuertemente centralizado y teniendo que buscar un difícil consenso, prefirieron no agotar el diseño territorial del Estado, que quedaba así en cierto modo "desconstitucionalizado", y dejar a los poderes constituidos la tarea de concluirlo. Así, la Constitución de 1978 no dio al Estado una forma políticamente descentralizada, sino que estableció las reglas para un proceso voluntario y gradual de descentralización. El sistema de distribución territorial del poder establecido en la Constitución es, por ello, un sistema abierto, que se ha ido construyendo de acuerdo con el denominado principio dispositivo. Por este motivo, el Consejo de Estado, antes de ocuparse de las cuestiones concretas que se propone analizar, realiza ciertas reflexiones sobre el principio dispositivo y la apertura del sistema autonómico que le servirán para contextualizar el análisis posterior de las mismas.

Así, el Consejo de Estado comienza recordando cómo «el principio dispositivo actúa de dos modos distintos, con objetivos diversos y en beneficio de sujetos diferentes" ${ }^{18}$.

En primer lugar, el principio dispositivo se manifiesta en aquellos preceptos que concedieron a las provincias la potestad de crear y organizarse en Comunidades Autónomas (arts. 143, 14, 148 y 151, y disps. transitorias 1. a a 7. ${ }^{\mathrm{a}} \mathrm{CE}$ ). A juicio del Consejo, estos preceptos son ya inaplicables desde que concluyó el proceso de creación de las Comunidades Autónomas y actualmente esta primera manifestación del principio dispositivo no tiene virtualidad alguna. Por eso, «bajo esa forma inicial el principio dispositivo desaparecerá de la Constitución con la derogación de los artículos que lo consagraron... derogación (que) no entraña, en puridad, consecuencia jurídica apreciable. (...) Por ello, la reforma constitucional proyectada se va a limitar a reflejar una realidad exis-

17 Ibíd., pp. 186 y 187.

18 Ibíd., p. 136. 
tente y, en esta forma concreta, el principio dispositivo no es ya parte de ella. El objetivo de superar la apertura inicial del modelo se tendrá que reducir, en consecuencia a dejar constancia de una superación ya producida, sin añadir nada a lo ya logrado" ${ }^{19}$.

Una vez creadas las Comunidades Autónomas, el principio dispositivo se manifiesta de otra manera, en la facultad de éstas de "redefinir de manera indefinida el ámbito de su autonomía", plasmada en los arts. 147.3 y 152.2 CE que regulan la reforma de los Estatutos, según los cuales "las Comunidades Autónomas pueden proponer y codecidir la modificación de sus propios ámbitos competenciales y alterar también, en cierto modo, el que queda en manos del Estado ${ }^{20}$. A juicio del Consejo de Estado, la eliminación de la forma inicial en que se manifestó el principio dispositivo obliga a reflexionar sobre los efectos que su subsistencia, en esa segunda forma, va a tener sobre la forma de organización territorial. "Las decisiones de eliminar una manifestación del principio dispositivo y mantener la otra están muy relacionadas entre sí. (...) esa estrecha relación autoriza, y aun obliga, a incluir en el Informe una reflexión sobre la apertura que se mantiene, a fin de sugerir, en su caso, las medidas necesarias para completar y perfeccionar técnicamente la reforma proyectada ${ }^{21}$. En este sentido el Consejo de Estado expone las siguientes reflexiones.

Para empezar, el Consejo afirma que, a diferencia de los Estados de estructura federal, en los que es la Constitución federal la que establece el ámbito competencial de los Estados federados, "la peculiaridad más acusada" de nuestra forma de organización territorial es que son los Estatutos de Autonomía los que establecen dicho ámbito competencial y, con ello, las competencias que quedan en manos del Estado central. Este sistema permite a las Comunidades Autónomas "tanto impulsar el cambio a través de la reforma de sus Estatutos, como impedirlo, vetando las que consideran inadecuadas". Esto, a juicio del Consejo, va más allá de la potestad de autoorganización que las Constituciones federales reconocen a los Estados miembros para la reforma de sus propias Constituciones, porque, aunque éstos pueden reformar su Constitución por sí mismos y la Federación sólo puede oponerse a ella impugnándola por infracción de los límites establecidos en la Constitución federal, las Constituciones de esos Estados tienen un alcance mucho menor que el de los Estatutos de Autonomía y no inciden sobre relaciones que están disciplinadas sólo por la Constitución federal ${ }^{22}$.

Siendo esto así, el Consejo recuerda la razón por la que en su momento se optó por el principio dispositivo: para originar un sistema diferenciado de distribución territorial del poder, según las necesidades de cada Autonomía, que se suponían diferentes en las distintas partes del territorio nacional; un sistema más diferenciado que el que cabría instaurar mediante la decisión directa del poder

19 Ibíd., p. 137.

20 Ibíd., p. 137.

21 Ibíd., p. 138.

22 Ibíd., p. 139. 
constituyente. Sin embargo, reconoce el Consejo que esa capacidad del principio dispositivo para crear un sistema diferenciado ha tropezado con dos obstáculos insalvables, de modo que hoy en día las diferencias organizativas entre Comunidades Autónomas son pequeñas y las de los respectivos ámbitos competenciales son casi todas transitorias. Por ello el Consejo considera que, si se tienen por superadas las circunstancias que llevaron a instaurarlo, las ventajas de este sistema abierto no son del todo evidentes ${ }^{23}$.

A juicio del Consejo, el primero de los obstáculos insalvables ha sido de carácter "objetivo" y se manifestó ya desde el primer momento. La conveniencia de que la reorganización de la Administración propia del Estado unitario y el traspaso a las Comunidades Autónomas de sus responsabilidades y recursos se hicieran de modo ordenado exigió el establecimiento de un calendario preciso y un diseño competencial dotado de un cierto grado de homogeneidad que el régimen de las "preautonomías" no aseguraba por sí mismo. Por ello, una vez que los tres territorios que podían acceder a la autonomía por la "vía rápida" lo hicieron, así como Andalucía, la libre iniciativa de las provincias para crear y definir nuevas Comunidades Autónomas quedó predeterminada por el acuerdo político alcanzado entre los dos partidos mayoritarios para evitar una excesiva heterogeneidad competencial entre dichas Comunidades. En los primeros acuerdos, los Pactos Autonómicos de 1981, se convino la aprobación de los Estatutos de esas comunidades con un contenido competencial semejante. Posteriormente, en 1992, se acordó que la reforma de dichos Estatutos, es decir, la ampliación de competencias, se produjese también de manera homogénea. Añade el Consejo que en ambos casos se hizo uso de un instrumento, las leyes orgánicas de transferencia y delegación, que parecía ajeno al principio dispositivo. En el primer caso, para transferir a algunas Comunidades Autónomas competencias que constitucionalmente aún no podían hacer suyas a través de los Estatutos; en el segundo, para poner a su disposición las competencias ofrecidas, antes de que éstas asumieran su titularidad mediante la reforma estatutaria ${ }^{24}$.

El segundo obstáculo, por el contrario, ha tenido un carácter más subjetivo. La creación de las Comunidades Autónomas avivó un "cierto espíritu de emulación, que empujaba a unos a luchar por el mantenimiento de una singularidad que en la Constitución era sólo temporal, y a los otros a oponerse a la concesión de mayores competencias o a reclamarlas también para sí. (...) Con el sólido apoyo del principio de igualdad, nuestras Comunidades Autónomas tienden por lo común a considerar que no deben existir entre los ámbitos competenciales respectivos más diferencias que aquellas que, como la lengua, los derechos forales o la insularidad, tienen reconocimiento explícito en la Constitución". Así, cualquier otra ampliación de competencias que consiga una Comunidad se convierte de inmediato en objetivo obligado a conseguir por todas las demás ${ }^{25}$. 
Por estos motivos concluye el Consejo que la ineficacia de este sistema abierto para alcanzar su objetivo, crear un sistema de distribución del poder diferenciado, hace más visibles los inconvenientes que se han puesto de relieve en la práctica. En relación a estos inconvenientes el Consejo señala que la reforma estatutaria es una posibilidad siempre abierta y es frecuente que los distintos partidos la incluyan en sus programas electorales, por regla general, para robustecer el poder de la respectiva Comunidad mediante la ampliación de sus competencias. Cuando la propuesta de reforma no surge de acuerdos generales, como los Pactos autonómicos anteriormente citados, el contraste entre la perspectiva particular (la de la Comunidad Autónoma) y la general (la de las Cortes Generales) ha de dilucidarse en el curso de la tramitación parlamentaria, de la que pueden surgir tensiones perjudiciales para la organización política y a veces también para la estabilidad de los partidos políticos. Estos riesgos se hacen más graves cuanto más se acerca el ámbito competencial de las Comunidades Autónomas al máximo admitido por la Constitución, ya que en ese caso cualquier propuesta de reforma que pretenda ampliar competencias puede ser acusada de pretender sobrepasar los límites constitucionales, y, con ello, «una cuestión jurídica se lleva al debate político, con daño tanto para el derecho como para la política". A lo que hay que añadir que el uso que las Comunidades Autónomas hacen de las facultades que el principio dispositivo les otorga está determinado, como es lógico, por los partidos políticos que las gobiernan $^{26}$.

Por todo esto concluye el Consejo de Estado que "no carecen de fundamento las dudas que desde hace algún tiempo se manifiestan acerca de la conveniencia de mantener en vigor un principio organizativo que no produce efectos que no pudieran lograrse, de manera jurídicamente más simple y políticamente menos traumática, por el simple procedimiento de llevar a la Constitución todo el sistema de delimitación de competencias. Este procedimiento es el común en el derecho comparado y, como bien muestra el ejemplo de nuestra propia Constitución, no implica la necesidad de una homogeneidad absoluta entre las Comunidades Autónomas, mientras que tampoco cierra a éstas la posibilidad de impulsar el cambio a través de la reforma constitucional que todas tienen la facultad de proponer". Reconoce el Consejo, no obstante, que "el abandono del principio dispositivo, poniendo término a la apertura del sistema, es una tarea compleja que no puede resolverse con fórmulas simplistas... (como) reducir el contenido competencial de todos los Estatutos a una simple norma que atribuyese todas las competencias que la Constitución no reserva en exclusiva al Estado, o modificar el apartado 3 del art. 149 de la Constitución". ${ }^{27}$

A pesar de haber manifestado su opinión en estos términos, el Consejo de Estado recuerda que «esa eventual reforma no es la que ahora se proyecta, circunscrita a cuestiones muy precisas, y cuyo propósito es el de superar la aper- 
tura inicial del sistema, no el de sustituir por otro el sistema abierto que hoy tenemos ${ }^{28}$.

\section{LOS LÍMITES A LA APERTURA, Y LAS LEYES DE TRANSFERENCIA Y DELEGACIÓN}

Así pues, la apertura de nuestro sistema de distribución territorial del poder se concreta ahora, creadas las Comunidades Autónomas, en la posibilidad de que dichas Comunidades reformen sus Estatutos. En la regulación constitucional de esta institución el Consejo de Estado distingue dos aspectos decisivos, «el procedimental y el material o sustancial: el procedimiento de reforma de los Estatutos y los límites que la Constitución impone a la ampliación de competencias $^{29}$. En cuanto a los procedimientos de reforma, en el Informe se analiza la variedad de procedimientos actualmente existente y se hacen ciertas reflexiones y propuestas de modificación de los mismos ${ }^{30}$.

Por lo que respecta a los límites a la apertura del sistema, esto es, los límites a las reformas estatutarias cuyo objeto es una ampliación de competencias, el Consejo señala que éstos son, en principio, los establecidos por las competencias exclusivas del Estado que enumera el art. 149 CE. No obstante, el art. $150 \mathrm{CE}$, que regula las leyes de trasferencia y delegación, crea una posibilidad de ir más allá de esos límites, aunque la ampliación de competencias que tiene lugar a través de estas leyes no es consecuencia del principio dispositivo ${ }^{31}$. En efecto, el art. 150.2 CE permite al Estado central «transferir o delegar en las Comunidades Autónomas, mediante ley orgánica, facultades correspondientes a materias de titularidad estatal que por su propia naturaleza sean susceptibles de transferencia o delegación", lo cual supone una vía extraordinaria de atribución de competencias a las Comunidades Autónomas, una vía "extraestatutaria" de ampliación competencial.

El Consejo recuerda que, como ponen de manifiesto los debates constituyentes, el objeto de prever este tipo de leyes fue el de introducir un elemento de flexibilidad o apertura en el sistema de distribución de competencias que facilitase su adaptación a nuevas circunstancias. Así pues, aunque los Estatutos alcancen el techo competencial establecido en la Constitución (todas las competencias no reservadas en exclusiva al Estado central por el art. 149.1), la distribución de competencias no puede considerarse cerrada definitivamente, puesto que siempre puede aprobarse una ley orgánica de transferencia o delegación que atribuya a las Comunidades Autónomas el ejercicio de nuevas competencias.

Por ello el Consejo considera que las citadas leyes forman parte de la apertura que caracteriza a nuestro sistema de distribución territorial del poder.

28 Ibíd., p. 144.

29 Ibíd., pp. 205 y 206.

30 Ibíd., pp. 206 a 218.

31 Ibíd., p. 218. 
"Su eliminación implicaría, por tanto, un cambio que va más allá de la proyectada reforma". Sin embargo, matiza el Consejo que "por el contrario, es perfectamente compatible con ella, y la haría técnicamente más perfecta, una modificación del precepto (el art. 150.2 CE) para hacerlo más claro y evitar las graves disfunciones derivadas de una utilización abusiva o masiva de este instrumento excepcional „32. Así pues, el Consejo termina proponiendo tres modificaciones concretas que afectarían al texto del art. 150.2 CE y que se corresponden con cuestiones que efectivamente han sido objeto de discusión doctrinal y no están definitivamente resueltas.

1. La primera modificación se refiere a la alternativa que ofrece la redacción actual del art. 150.2, "transferencia o delegación". El Consejo de Estado considera que se conseguiría mayor claridad simplificando el texto actual del art 150.2 CE en dos sentidos. De un lado, el texto se refiere en dos ocasiones a la transferencia y la delegación como opciones alternativas, lo que ha creado cierta confusión perturbadora. El Consejo afirma que, siendo mayoritaria entre los especialistas la opinión de que no existen diferencias cualitativas que justifiquen tal disyuntiva, puesto que en todos los casos es el ejercicio y no la titularidad de las facultades lo que se cede a las Comunidades Autónomas, no tiene mucho sentido mantener esta dualidad. De otro, "para disipar cualquier duda al respecto, también convendría precisar que lo que se cede por este procedimiento, a una o varias Comunidades Autónomas, es únicamente el ejercicio". Así, la redacción del art. 150.2 CE podría ser la siguiente: "El Estado podrá transferir a una o varias Comunidades Autónomas el ejercicio de facultades sobre materias de titularidad estatal, ${ }^{33}$.

La formulación del art. 150.2 CE es ciertamente confusa, ya que al utilizar dos términos diferentes, y que en derecho público tienen un significado distinto, parecen consagrarse dos cauces alternativos; aunque, por otro lado, al someterlos a previsiones comunes puede entenderse también que la Constitución asimila ambas nociones. Así no es de extrañar que la doctrina haya estado dividida a la hora de interpretar si estamos ante una o dos posibilidades diferentes, y cuál es el significado de cada una de ellas (si la transferencia lo es de la titularidad, y la delegación simplemente del ejercicio; si aquélla no admite revocación, mientras que la delegación sí...). Aunque actualmente se reconoce la dificultad de distinguir dos técnicas en el art. 150.2 CE coincidentes con las nociones de delegación y transferencia acuñadas por la doctrina iuspublicista clásica, lo cierto es que la doctrina constitucional no deja de admitir que la existencia de dos posibilidades permite pensar en diferentes matices, distintos grados de intensidad en la traslación de competencias a las Comunidades Autónomas, como, por otra parte, ha sido la práctica hasta ahora ${ }^{34}$.

32 Ibíd., p. 219.

33 Ibíd., pp. 219 y 220.

34 Sirvan como referencia de las distintas interpretaciones los trabajos de J. A. MONTILLA, Las leyes orgánicas de transferencia y delegación, 1998, pp. 133 y ss.; C. VIVER, "Transferencia y delegación de competencias del Estado a las Comunidades Autónomas", en Enciclopedia Jurídica Bá- 
2. En segundo lugar, el Consejo considera que "convendría delimitar el ámbito de las facultades de titularidad estatal que pueden ser delegadas mediante algún criterio más preciso que la naturaleza de éstas, que es el que el texto actual utilizam. Efectivamente, en el debate doctrinal sobre las leyes de transferencia y delegación otra de las cuestiones centrales, si no la central, es la determinación de las facultades y las materias que pueden ser transferidas o delegadas a las Comunidades Autónomas. Aquí el texto constitucional tampoco da una respuesta concluyente y en la doctrina nos encontramos igualmente con diferentes interpretaciones al respecto. Para resolver esta situación el Consejo ofrece dos vías concurrentes.

La primera, caracterizar las facultades que en ningún caso pueden ser delegadas (o transferidas). Esta caracterización debería hacerse en términos muy genéricos y debería incluir, además de las que se consideren inherentes al ejercicio de la soberanía e indispensables para asegurar el correcto funcionamiento de la organización general del Estado, las que pongan en peligro la capacidad del Gobierno para diseñar y aplicar su propia política en aquellos ámbitos que la Constitución le ha reservado en exclusiva (facultades de dirección política, según el art. 97)». Formaría parte también "de este núcleo duro intangible la competencia atribuida al Estado en numerosos apartados del art. 149.1 para dictar las bases o la legislación básica; una competencia para definir el denominador común normativo que, en buena lógica, no puede ser cedida a una Comunidad Autónoma ${ }^{35}$.

La segunda, "para precisar igualmente cuáles son las facultades delegables o transferibles desde el punto de vista de la finalidad perseguida por la delegación o transferencia, exigir, como requisito adicional, que se trate de facultades necesarias para que la Comunidad ejerza más eficazmente las competencias ya asumidas en sede estatutaria, con exclusión, por tanto, de todas las que se proyecten sobre materias en las que la Comunidad Autónoma no puede invocar ningún título que la habilite de una u otra forma para intervenir". Añade el Consejo que "la exigencia de una vinculación directa entre una o varias competencias de las que figuran en el Estatuto como propias de la Comunidad Autónoma y las que eventualmente pudieran traspasarse por el procedimiento previsto en el art. 150.2 serviría para poner coto a la fuerza expansiva de esta técnica, sometiendo su uso a una condición que se formula en términos positivos ${ }^{36}$.

3. En tercer lugar, el Consejo concluye que "sería conveniente aprovechar la reforma para zanjar definitivamente la polémica en torno a la utilización de los Estatutos de Autonomía como instrumentos para formalizar la cesión de competencias al amparo de la habilitación contenida en el art. 150.2, aunque

sica, vol. IV, pp. 6626 y ss.; E. AJA, "La modificación extraestatutaria de las competencias", en AAVV, El sistema jurídico de las Comunidades Autónomas, 1985, pp. 173 y ss.; M. BASSOLS Y J. M. SERRANO, "El art. 149 de la Constitución en relación al 150.2", RAP n. ${ }^{\circ}$ 97, 1982, pp. 58 y 59.

35 Consejo de Estado, Informe sobre modificaciones de la Constitución Española, p. 220.

36 Ibíd., pp. 220 y 221. 
esta utilización se haga ya difícil si, como antes se propugna, se rompe la conexión entre Estatutos de Autonomía y leyes orgánicas. Apelando a la rotunda jurisprudencia constitucional y a la interpretación dominante en la doctrina científica, podría incluirse una expresa interdicción de la incorporación al texto estatutario de estas facultades estatales trasferidas $" 37$.

Finalmente, en relación a estas propuestas para modificar el art. 150.2 CE y completar así la reforma proyectada, debemos hacer referencia a la existencia de dos votos particulares que se presentaron al Informe aprobado por el pleno del Consejo y en los que se hace referencia a esta propuesta; en un caso, apoyándola, y, en el otro, yendo más lejos y propugnando incluso la derogación del art. $150.2 \mathrm{CE}^{38}$.

ABSTRACT. This paper analyses the Report on the Spanish Constitution's Reform drafted by the Council of State (Consejo de Estado). It focus on the discussion about the reform to include in the Constitution the name of the Regions, the degree of flexibility of our territorial system of government, and the role played by the leyes orgánicas de transferencia y delegación, laws regulated in the art. 150.2 of the Spanish Constitution that can be approved by the central parliament to delegated central powers in some matters to the Regions.

37 Ibíd., p. 221.

38 En el primero, formulado por D. Luis Díez-Picazo, éste afirma que «en términos generales estoy a favor de mantener las competencias del Estado, por supuesto, y en reducir el alcance de la posibilidad de trasferencias del art. 150" (p. 358). En el segundo, formulado por D. José M. Aznar, éste comparte la identificación que hace el Consejo de los problemas del sistema abierto de distribución territorial del poder y la necesidad de reconsiderar el principio dispositivo, y, en relación a las leyes de trasferencia y delegación, afirma que coincide "en buena medida, con las consideraciones del Informe sobre el art. 150.2. A mi juicio, se debería contemplar como posible opción su derogación. Dicho mecanismo ha dado de sí lo que razonablemente podía justificar su introducción en la Constitución. En mi opinión, carece de sentido mantener cláusulas que tiene por efecto desconstitucionalizar parcialmente el modelo de Estado o estimular la dinámica de reivindicación desde las Comunidades Autónomas sobre las competencias estatales. Derogar la previsión del art. 150.2 significa, por tanto, que futuros aumentos del acervo competencial de las Comunidades Autónomas requerirían una reforma constitucional" (p. 367). 